\title{
Hierarchical service restoration scheme for active distribution networks based on ADMM
}

Shen, Feifan; Wu, Qiuwei; Xu, Yan; Li, Fangxing; Teng, Fei; Strbac, Goran

Published in:

International Journal of Electrical Power and Energy Systems

Link to article, DOI:

10.1016/j.ijepes.2019.105809

Publication date:

2019

Document Version

Peer reviewed version

Link back to DTU Orbit

Citation (APA):

Shen, F., Wu, Q., Xu, Y., Li, F., Teng, F., \& Strbac, G. (2019). Hierarchical service restoration scheme for active distribution networks based on ADMM. International Journal of Electrical Power and Energy Systems, 118, [105809]. https://doi.org/10.1016/j.ijepes.2019.105809

\section{General rights}

Copyright and moral rights for the publications made accessible in the public portal are retained by the authors and/or other copyright owners and it is a condition of accessing publications that users recognise and abide by the legal requirements associated with these rights.

- Users may download and print one copy of any publication from the public portal for the purpose of private study or research.

- You may not further distribute the material or use it for any profit-making activity or commercial gain

- You may freely distribute the URL identifying the publication in the public portal 


\title{
Hierarchical Service Restoration Scheme for Active Distribution Net- works based on ADMM
}

\author{
Feifan Shen a ${ }^{\text {a }}$ Qiuwei Wu ${ }^{\text {a, }}$, Yan Xu ${ }^{\text {b }}$, Fangxing $\mathrm{Li}^{\mathrm{c}}{ }^{\text {, Fei Teng }}{ }^{\mathrm{d}}$, Goran Strbac ${ }^{\mathrm{d}}$ \\ ${ }^{a}$ Centre for Electric Power and Energy, Department of Electrical Engineering, Technical University of Denmark, Kgs. Lyngby, DK 2800 \\ ${ }^{\mathrm{b}}$ School of Electrical and Electronic Engineering, Nanyang Technological University, 50 Nanyang Avenue, Singapore, 639798 \\ ${ }^{\mathrm{c}}$ Department of Electrical Engineering and Computer Science, University of Tennessee, Knoxville, TN, USA, 37996 \\ d Department of Electrical and Electronic Engineering, Imperial College London, South Kensington Campus, London SW7 2AZ, UK \\ * Corresponding author: e-mail: qw@elektro.dtu.dk
}

\begin{abstract}
Effective self-healing schemes enhance the resilience of active distribution networks (ADNs). As a critical part of selfhealing, service restoration aims to restore outage areas with minimal un-supplied demands. With the increasing complexity and size of ADNs, distribution system operators (DSOs) face a more complicated service restoration problem. Thus, it is important to obtain optimal service restoration plans and reduce computational complexity. To achieve this goal, a hierarchical service restoration scheme is proposed to obtain service restoration plans based on the alternating direction method of multipliers (ADMM). The optimal service restoration problem is formulated as a mixed-integer linear programming (MILP) model considering the switching sequence, distributed generation (DG) units and controllable loads, and is solved using the ADMM-based algorithm in a hierarchical manner. In the proposed scheme, each zone of the ADN has a local service restoration controller solving its sub-problem with information from a central service restoration controller. The central controller solves a global coordination problem with information from all the zones. Three case studies were conducted with the 44-node test system, modified IEEE 123-node system and Brazil 948-node system. The results show that the proposed hierarchical service restoration can obtain optimal service restoration plans and reduce computational complexity. Moreover, computation time can be reduced substantially by using the proposed hierarchical scheme for large-scale ADNs.
\end{abstract}

Keywords: Active distribution networks, alternating direction method of multipliers, hierarchical service restoration, self-healing

\section{Nomenclature}

Sets and indices:

$\mathcal{N}, \mathcal{N}^{S}$ Set of nodes and set of main source nodes

$\mathcal{L} \quad$ Set of branches and switches

$\mathcal{L}^{B}, \mathcal{L}^{\mathcal{S}} \quad$ Set of branches and set of switches

$\mathcal{L}^{F} \quad$ Set of switches connected to the faulted zone

$\mathcal{Z} \quad$ Set of zones

$\mathcal{S} \quad$ Set of restoration steps, i.e., $s_{1}, s_{2}, . ., s_{\max }$

$\mathcal{B}(z) \quad$ Set of nodes belonging to zone $z \in \mathcal{Z}$
$\mathcal{P} \quad$ Set of discrete segments

|*| Cardinality of sets, e.g., $|\mathcal{N}|,\left|\mathcal{N}^{s}\right|,|\mathcal{P}|$

$i, j, z \quad$ Nodes $i$ and $j \in \mathcal{N}$; zone $z \in \mathcal{Z}$

$s \quad$ Step $s \in \mathcal{S}$

Variables:

$P_{i, s}^{j}, Q_{i, s}^{j} \quad$ Active and reactive power flow from node $i$ to node $j$ at step $s$ [MW, MVar] 
$H_{i, s}^{j} \quad$ Fictitious active power flow from node $i$ to node $j$ at $\quad c_{i}^{\mathrm{r}}$ step $s[\mathrm{MW}]$

$P_{i, s}^{\mathrm{G}}, Q_{i, s}^{\mathrm{G}} \quad$ Active and reactive DG generations at node $i$ at step $s[\mathrm{MW}, \mathrm{MVar}]$

$H_{i, s}^{\mathrm{G}} \quad$ Fictitious active power generation at node $i$ at step $s$ [MW]

$I_{i, s}^{j, s q r} \quad$ Square current magnitude from node $i$ to node $j$ at step $s\left[\mathrm{kA}^{2}\right]$

$V_{i, s}^{\text {sqr }} \quad$ Square voltage magnitude at node $i$ at step $s\left[\mathrm{kV}^{2}\right]$

$r_{i, s} \quad$ Percentage of load shedding at node $i$ at step $s$

$y_{i, s}^{j, \mathrm{u}}, y_{i, s}^{j, \mathrm{~d}}$ Auxiliary binary variables associated with switching

$w_{i, s}^{j} \quad$ Status of the switch between nodes $i$ and $j$ at step $s$, where $w_{i, s}^{j}=1$ if the switch is closed at step $s$; otherwise, $w_{i, s}^{j}=0$

$x_{z, s} \quad$ Status of zone $z$ at step $s$, where $x_{z, s}=1$ if the zone is energized at step $s$; otherwise, $x_{z, s}=0$

$z^{+}, z^{-}, \Delta z$ Auxiliary variables used to linearize $z^{2}$.

$\bar{z} \quad$ Maximum value of variable $z$

Parameters:

$c_{i}^{\mathrm{G}} \quad$ Cost of generation at node $i[\mathrm{~m} . \mathrm{u} . / \mathrm{kW}]$

$c_{i}^{j, \text { lss }} \quad$ Cost of active power losses of the circuit between nodes $i$ and $j[$ m.u./kW]

$c_{i}^{j, \mathrm{~s}} \quad$ Cost of a switching action of the switch between nodes $i$ and $j$ [m.u.]
$c_{z}^{\mathrm{U}} \quad$ Cost of de-energizing zone $z: c_{i}^{\mathrm{r}} P_{i}^{\mathrm{D}}, i \in \mathcal{B}(z)[$ m.u.]

$\hat{z}_{i} \quad$ The index of the zone to which node $i$ belongs

$\bar{I}_{i}^{j, \mathrm{sqr}} \quad$ Maximum square current magnitude of the circuit between nodes $i$ and $j\left[\mathrm{kA}^{2}\right]$

$\bar{H}_{i}^{j} \quad$ The upper bound of fictitious active power flow from node $i$ to node $j$ [MW]

$\bar{P}_{i}^{\mathrm{G}}, \bar{Q}_{i}^{\mathrm{G}} \quad$ Maximum active and reactive DG generations at node $i$ [MW, MVar]

$P_{i}^{\mathrm{D}}, Q_{i}^{\mathrm{D}} \quad$ Active and reactive demands at node $i$ [MW, MVar]

$K_{i}^{\mathrm{D}} \quad$ Fictitious active demand at node $i[\mathrm{MW}]$

$R_{i}^{j}, X_{i}^{j} \quad$ Resistance and reactance of the circuit between nodes $i$ and $j$

$\bar{V}^{\text {sqr }}, \underline{V}^{\text {sqr }}$ Maximum and minimum square voltage magnitude $\left[\mathrm{kV}^{2}\right]$

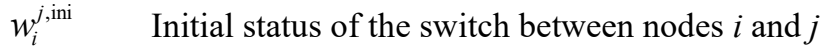

$n_{l} \quad$ The number of branches

$\varepsilon_{1}, \varepsilon_{2} \quad$ Specified thresholds for primal and dual residuals

$\rho \quad$ The penalty parameter of the ADMM-based algorithm

$\mu, \tau \quad$ Parameters for tuning the penalty parameter $\rho$

\section{Introduction}

An essential aspect of reliable operation of active distribution networks (ADNs) is to efficiently cope with emergency events [1]. The rapid development of the distribution automation (DA) and advanced metering infrastructure (AMI) provides ADNs self-healing capability. After a permanent fault occurs, the self-healing scheme detects the fault location, isolates the faulted portion and restores the network with minimum un-supplied demands based on a set of DA devices, algorithms and communication technologies [2]. Self-healing schemes benefit ADNs in both functional and monetary aspects [3]. For the functional benefits, it improves the reliability indexes such as the system average interruption duration index (SAIDI). For the monetary benefits, it ensures continuity of power supply and reduces the cost of un-supplied demands. 
The self-healing scheme should provide effective service restoration plans with limited computational resources [4]. Service restoration plans are obtained using expert systems in [5], [6] and heuristic algorithms in [7], [8]. The multi-agent systems (MASs) are also widely used to solve service restoration problems [9]-[12]. In the MAS, agents communicate and coordinate to obtain restoration plans using heuristic rules. The above approaches use problem-dependent heuristics to search for solutions. Without requiring problem-dependent heuristics, meta-heuristic algorithms were applied in [13] and [14] to solve service restoration problems. By treating the ADN as a graph, graph theory-based approaches [15], [16] were developed to find a radial configuration of the network without violating operational constraints. Most of the above approaches obtain local solutions.

Mathematical programming based approaches [17]-[23] have been proposed to obtain optimal or sub-optimal service restoration plans from the system perspective. Two mixed-integer non-linear programming (MINLP) models were developed in [17] for service restoration based on the branch flow formulation and bus injection formulation, respectively. A two-stage service restoration strategy was proposed in [18], in which the configuration of the network is determined in the first stage and the operating point is adjusted in the second stage. Mixed-integer linear programming (MILP) models of service restoration considering the switching sequence were developed in [19] and [20]. Robust service restoration models were developed in [21] and [22] to deal with uncertainties of renewable energy resources (RESs) and loads. The above approaches can obtain optimal service restoration plans. However, they solve service restoration problems in a centralized manner and have high computational complexity for largescale ADNs. An alternating direction method of multipliers (ADMM) based distributed service restoration strategy was developed in [23], in which the service restoration problem is solved using multiple agents. However, the switching operation as an important option to perform service restoration is not considered.

To resolve the above issues, an ADMM-based hierarchical service restoration scheme for ADNs is proposed to obtain the optimal service restoration plan in a hierarchical manner. Firstly, the optimal service restoration problem is formulated as a MINLP model considering the switching sequence, distributed generation (DG) units and controllable loads. Then, the MINLP model is transformed into a MILP model by using linearization techniques, which is decomposed and solved using the proposed ADMMbased algorithm in a hierarchical manner.

The contributions of this paper are twofold: 1) propose a MILP model of service restoration for ADNs considering the switching sequence, DG units and controllable loads; 2) develop an ADMM-based hierarchical solution method to obtain optimal service restoration plans and reduce computational complexity.

The paper is organized as follows: Section II introduces the framework of the proposed hierarchical service restoration scheme. Section III presents the MINLP and MILP models of service restoration for ADNs. Section IV presents the proposed ADMM-based solution method. Case studies are provided in Section V, followed by the conclusions.

\section{Framework of the Proposed Hierarchical service restoration Scheme}

In this paper, the service restoration problem is solved using a hierarchical framework shown in Fig. 1. The proposed framework is based on independent local controllers and a central controller with limited information exchanged among them. As shown in Fig. 1, a zone is an interconnected portion of the network separated by switches and has a local controller. Based on the ADMM-based algorithm, each zone controller solves its own sub-problem with local network data and information from the central controller to optimize variables associated with the zone, e.g., the amount of load shedding. The central controller solves a global problem with information collected from each zone to coordinate the status of switches and zones. 


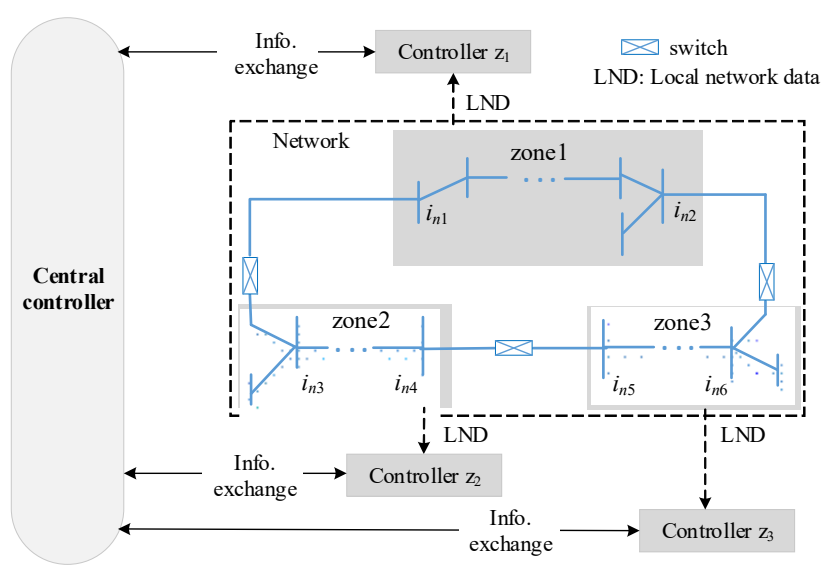

Fig. 1. Framework of the proposed hierarchical service restoration scheme.

\section{Problem Formulation for Optimal Service Restoration}

\subsection{Optimal Service Restoration Model}

Consider the $\mathrm{ADN}$ as a directed graph $\mathcal{G}=(\mathcal{N}, \mathcal{L})$, where $\mathcal{N}$ denotes the set of nodes and $\mathcal{L}$ denotes the set of branches and switches. In the $\mathrm{ADN}$, every node and branch belong to a unique zone $z \in \mathcal{Z}$ that is a portion of the network separated by switches. The optimal service restoration problem for the ADN is formulated as a MINLP model shown in (1)-(16).

3.1.1 Objective function

$$
\begin{aligned}
\min & \sum_{z \in \mathcal{Z}} \sum_{s \in \mathcal{S}} c_{z}^{\mathrm{U}}\left(1-x_{z, s}\right)+\sum_{i \in \mathcal{N}} \sum_{s \in \mathcal{S}} c_{i}^{\mathrm{G}} P_{i, s}^{\mathrm{G}}+\sum_{i \in \mathcal{N}} \sum_{s \in \mathcal{S}} c_{i}^{\mathrm{r}} r_{i, s} P_{i}^{\mathrm{D}} \\
& +\sum_{(i, j) \in \mathcal{L}} \sum_{s \in \mathcal{S}} c_{i}^{j, \mathrm{lss}} I_{i, s}^{j, \mathrm{sqr}} R_{i}^{j}+\sum_{(i, j) \in \mathcal{L}^{\mathcal{S}}} \sum_{s \in \mathcal{S}} c_{i}^{j, \mathrm{~s}}\left(y_{i, s}^{j, \mathrm{u}}+y_{i, s}^{j, \mathrm{~d}}\right)
\end{aligned}
$$

The objective function in (1) minimizes five terms during service restoration: the number of de-energized zones, energy production cost, cost of load shedding, cost of line losses and number of switching operations.

\subsubsection{Constraints}

$$
\begin{aligned}
& P_{i, s}^{\mathrm{G}}+\sum_{(j, i) \in \mathcal{L}} P_{j, s}^{i}-\sum_{(i, j) \in \mathcal{L}}\left(P_{i, s}^{j}+I_{i, s}^{j, \mathrm{sqr}} R_{i}^{j}\right)=P_{i, s}^{\mathrm{D}}\left(x_{z, s}-r_{i, s}\right) ; \forall z \in \mathcal{Z}, i \in \mathcal{B}(z), s \in \mathcal{S} \\
& Q_{i, s}^{\mathrm{G}}+\sum_{(j, i) \in \mathcal{L}} Q_{j, s}^{i}-\sum_{(i, j) \in \mathcal{L}}\left(Q_{i, s}^{j}+I_{i, s}^{j, \mathrm{sqr}} X_{i}^{j}\right)=Q_{i, s}^{\mathrm{D}}\left(x_{z, s}-r_{i, s}\right) ; \quad \forall z \in \mathcal{Z}, i \in \mathcal{B}(z), s \in \mathcal{S} \\
& 0 \leq r_{i, s} \leq x_{z, s} ; \quad \forall z \in \mathcal{Z}, i \in \mathcal{B}(z), s \in \mathcal{S} \\
& V_{i, s}^{\mathrm{sqr}}-V_{j, s}^{\mathrm{sqr}}=2\left(P_{i, s}^{j} R_{i}^{j}+Q_{i, s}^{j} X_{i}^{j}\right)+I_{i, s}^{j, \mathrm{gqr}}\left[\left(R_{i}^{j}\right)^{2}+\left(X_{i}^{j}\right)^{2}\right] ; \quad \forall(i, j) \in \mathcal{L}^{B}, s \in \mathcal{S} \\
& -\bar{V}^{\mathrm{sqr}}\left(1-w_{i}^{j}\right) \leq V_{i, s}^{\mathrm{sqr}}-V_{j, s}^{\mathrm{sqr}} \leq \bar{V}^{\mathrm{sqr}}\left(1-w_{i}^{j}\right) ; \quad \forall(i, j) \in \mathcal{L}^{S}, s \in \mathcal{S} \\
& V_{j, s}^{\mathrm{sqr}} I_{i, s}^{j, \mathrm{sqr}}=\left(P_{i, s}^{j}\right)^{2}+\left(Q_{i, s}^{j}\right)^{2} ; \quad \forall(i, j) \in \mathcal{L}, s \in \mathcal{S} \\
& x_{z, s} V^{\mathrm{sqr}} \leq V_{i, s}^{\mathrm{sqr}} \leq x_{z, s} \bar{V}^{\mathrm{sqr}} ; \quad \forall z \in \mathcal{Z}, i \in \mathcal{B}(z), s \in \mathcal{S} \\
& \left\{\begin{array}{l}
I_{i, s}^{j, \mathrm{sqr}} \leq x_{z, s} \bar{I}_{i}^{j, \mathrm{sqr}} ; \quad \forall z \in \mathcal{Z},(i, j) \in \mathcal{L}^{B}, s \in \mathcal{S} \\
I_{i, s}^{j, \mathrm{sqr}} \leq w_{i, s}^{j} \bar{I}_{i}^{j, \mathrm{sqr}} ; \quad \forall(i, j) \in \mathcal{L}^{S}, s \in \mathcal{S}
\end{array}\right.
\end{aligned}
$$




$$
\begin{aligned}
& \left\{\begin{array}{l}
0 \leq P_{i, s}^{\mathrm{G}} \leq \bar{P}_{i}^{\mathrm{G}} x_{\hat{z}_{i}, s} ; \quad \forall i \in \mathcal{N}, s \in \mathcal{S} \\
0 \leq Q_{i, s}^{\mathrm{G}} \leq \bar{Q}_{i}^{\mathrm{G}} x_{\hat{z}_{i}, s} ; \quad \forall i \in \mathcal{N}, s \in \mathcal{S}
\end{array}\right. \\
& w_{i, s}^{j}-w_{i, s-1}^{j}=y_{i, s}^{j, \mathrm{u}}-y_{i, s}^{j, \mathrm{~d}} ; \quad \forall(i, j) \in \mathcal{L}^{\mathcal{S}}, s \in \mathcal{S} \mid w_{i, s_{1}-1}^{j}=w_{i}^{j, \mathrm{ini}} \\
& \sum_{(i, j) \in \mathcal{L}^{\mathcal{S}}}\left(y_{i, s}^{j, \mathrm{u}}+y_{i, s}^{j, \mathrm{~d}}\right) \leq 1 ; \quad \forall s \in \mathcal{S} \\
& \left|x_{\hat{\hat{z}}_{i}, s}-x_{\hat{\hat{z}}_{j}, s}\right| \leq 1-w_{i, s}^{j} ; \quad \forall(i, j) \in \mathcal{L}^{S}, s \in \mathcal{S} \\
& \sum_{(i, j) \in \mathcal{L}^{\mathcal{S}}} w_{i, s}^{j} x_{\hat{z}_{i}, s}=\sum_{z \in \mathcal{Z}} x_{z, s} ; \quad \forall s \in \mathcal{S} \\
& H_{i, s}^{\mathrm{G}}+\sum_{(j, i) \in \mathcal{L}} H_{j, s}^{i}-\sum_{(i, j) \in \mathcal{L}} H_{i, s}^{j}=K_{i}^{\mathrm{D}} x_{z, s} ; \forall z \in \mathcal{Z}, i \in \mathcal{B}(z), s \in \mathcal{S} \\
& \begin{cases}H_{i, s}^{j} \leq x_{z, s} \bar{H}_{i}^{j} ; & \forall z \in \mathcal{Z},(i, j) \in \mathcal{L}^{B}, s \in \mathcal{S} \\
H_{i, s}^{j} \leq w_{i, s}^{j} \bar{H}_{i}^{j} ; & \forall(i, j) \in \mathcal{L}^{S}, s \in \mathcal{S}\end{cases} \\
& H_{i, s}^{\mathrm{G}}=0 ; \forall i \in \mathcal{N} \backslash \mathcal{N}^{S}, s \in \mathcal{S} \\
& \left|w_{i, s+1}^{j}-w_{i, s}^{j}\right| \leq 1-w_{i^{*}, s}^{j^{*}} ; \quad \forall\left(i^{*}, j^{*}\right) \in \mathcal{L}^{F},(i, j) \in \mathcal{L}^{S} \backslash \mathcal{L}^{F}, s \in \mathcal{S} \\
& x_{z, s}, w_{i, s}^{j}, y_{i, s}^{j, \mathrm{u}}, y_{i, s}^{j, \mathrm{~d}} \in\{0,1\} ; \quad \forall z \in \mathcal{Z},(i, j) \in \mathcal{L}^{S}, s \in \mathcal{S}
\end{aligned}
$$

Constraints (2) and (3) represent the active and reactive power balance at each node at each step, respectively. Constraint (4) limits the percentage of load shedding of each node at each step. The voltage drop at each branch at each step is calculated in (5). Constraint (6) ensures that if a switch is closed, voltage magnitudes at both ends of the switch are identical. Current magnitudes of all branches and switches at each step are calculated in (7). Constraint (8) limits voltage magnitudes of all nodes at each step: if a given zone is de-energized, voltage magnitudes of all nodes in the zone are forced to be zero. Constraint (9) limits the square current magnitudes of branches and switches at each step. Constraint (10) limits the maximum active and reactive DG generations at each node at each step. Constraint (11) models the switching action. Constraint (12) ensures at most one switching operation at each step. Constraint (13) represents that connected zones must have the same status. Constraints (14) and (15) ensure a radial topology of the energized portion of the network at each step [24]. Constraints (15)-(17) force energized zones being connected to the main grid by supplying fictitious active demands of each energized zone from substations. Constraint (18) represents that switches not connected to the faulty zone can operate only after the switches connected to the faulty are opened, denoting that the service restoration process starts after the isolation of the faulty zone. The binary decision variables are defined in (19).

\subsection{Linearization of the MINLP Model}

The optimal service restoration problem formulated as a MINLP model is hard to solve. To efficiently obtain service restoration plans, linearization techniques are used to linearize non-linear constraints (7) and (14), which results in a MILP model.

\subsubsection{Linearization of (7)}

Firstly, the product of continuous variables $V_{j, s}^{\mathrm{sqr}} I_{i, s}^{j, \mathrm{sqr}}$ in (7) is approximated in (20) by fixing $V_{j, s}^{\mathrm{sqr}}$ as the square nominal value $\left(V^{\text {nom }}\right)^{2}$. The approximation error is low because voltage magnitudes are constrained in a small range. Secondly, the square terms in (7) are approximated using the piece-wise linearization technique [18] as shown in (21)-(22).

$$
V_{j, s}^{\mathrm{sqr}} I_{i, s}^{j, \mathrm{sqr}} \approx\left(V^{\mathrm{nom}}\right)^{2} I_{i, s}^{j, \mathrm{sqr}}
$$




$$
\left(P_{i, s}^{j}\right)^{2} \approx L\left(P_{i, s}^{j}\right),\left(Q_{i, s}^{j}\right)^{2} \approx L\left(Q_{i, s}^{j}\right) ;
$$

where function $L(z)$ is defined as follows,

$$
\begin{aligned}
& L(z)=\sum_{\lambda=1}^{\mid \mathcal{P}} \phi_{\lambda} \Delta z_{\lambda} ; \\
& \sum_{\lambda=1}^{\mid \mathcal{P}} \Delta z_{\lambda}=z^{+}+z^{-} ; \\
& z=z^{+}-z^{-} ; \\
& 0 \leq \Delta z_{\lambda} \leq \bar{z} /|\mathcal{P}| ; \quad \forall \lambda \in \mathcal{P} \\
& \phi_{\lambda}=(2 \lambda-1)^{\bar{z}} /|\mathcal{P}| ; \forall \lambda \in \mathcal{P} \\
& z^{+}, z^{-} \geq 0
\end{aligned}
$$

Then, the linearization approximation of (7) is obtained as,

$$
\left(V^{\mathrm{nom}}\right)^{2} I_{i, s}^{j, \mathrm{sqr}}=L\left(P_{i, s}^{j}\right)+L\left(Q_{i, s}^{j}\right) ; \quad \forall(i, j) \in \mathcal{L}, s \in \mathcal{S}
$$

\subsubsection{Linearization of (14)}

The product of binary variables $w_{i, s}^{j} x_{\hat{z}_{i}, S}$ in (14) is replaced by a continuous auxiliary variable $\tau_{i, s}^{j}$ as shown in (24).

$$
\left\{\begin{array}{l}
\sum_{(i, j) \in \mathcal{L}^{\mathcal{S}}} \tau_{i, s}^{j}=\sum_{z \in \mathcal{Z}} x_{z, s} ; \quad \forall s \in \mathcal{S} \\
w_{i, s}^{j}+x_{\hat{z}_{i}, s}-1 \leq \tau_{i, s}^{j} ; \quad \forall(i, j) \in \mathcal{L}^{S}, s \in \mathcal{S} \\
0 \leq \tau_{i, s}^{j} \leq w_{i, s}^{j} ; \quad \forall(i, j) \in \mathcal{L}^{S}, s \in \mathcal{S} \\
0 \leq \tau_{i, s}^{j} \leq x_{\hat{z}_{i}, s} ; \quad \forall(i, j) \in \mathcal{L}^{S}, s \in \mathcal{S}
\end{array}\right.
$$

Finally, the MILP model is formulated as (1)-(6), (8)-(13), (15)-(19) and (23)-(24).

\section{ADMM Based Algorithm for the MILP Model}

As a distributed optimization technique, the ADMM has been widely used to distribute and solve convex optimization problems. In [25], an ADMM-based algorithm was developed to solve multi-area economic dispatch problems in a fully distributed manner and was compared with two widely used distributed algorithms including the Lagrangian relaxation and auxiliary problem principle. In [26], an ADMM-based distributed residential direct load control strategy was proposed to adjust the residential electricity demand for peak load shaving with privacy information protection. In [27], a distributed reactive power control scheme based on the consensus ADMM was designed for voltage control of the wind farm cluster, which can achieve fair reactive power sharing among wind farms to regulate bus voltages. Although the ADMM is originally used to solve convex problems, recent studies show that it is effective in obtaining high-quality solutions for non-convex problems [28] such as unit commitment problems [29]. In this section, the ADMM-based algorithm is used to distribute and solve the MILP model in a hierarchical fashion and information exchanges in the hierarchical framework are illustrated.

4.1 Reformulation of the MILP Model for the ADMM

For node $i \in \mathcal{N}$ at step $s \in \mathcal{S}$, define sets $\mathcal{P}_{i, s}, \mathcal{I}_{i, s}$ and $\mathcal{W}_{i, s}$ as below, 


$$
\left\{\begin{array}{l}
\mathcal{P}_{i, s}:=\left\{\left\{P_{i, s}^{j}, H_{i, s}^{j}, Q_{i, s}^{j}\right\}_{(i, j) \in \mathcal{L}}, V_{i, s}^{\mathrm{sgr}}\right\} ; \\
\mathcal{I}_{i, s}:=\left\{\left\{I_{i}^{j, \mathrm{sgr}}\right\}_{(i, j) \in \mathcal{L}}, r_{i}, P_{i}^{\mathrm{G}}, Q_{i}^{\mathrm{G}}\right\} ; \\
\mathcal{W}_{i, s}:=\left\{\left\{w_{i, s}^{j}, y_{i}^{j, \mathrm{~d}}, y_{i}^{j, \mathrm{u}}, \tau_{i, s}^{j}\right\}_{(i, j) \in \mathcal{L}^{s}}\right\} ;
\end{array}\right.
$$

Variables in $\mathcal{P}_{i, s}, \mathcal{I}_{i, s}$ and $\mathcal{W}_{i, s}$ are associated variables of node $i$. Since each node belongs to a unique zone, variables with the subscript $i$ are exclusively optimized by the corresponding zone controller $\hat{z}_{i}$. For clarity, the MILP model is presented in a compact form.

The objective function in (1) is separable for each zone and is rewritten as,

$$
\min f\left(\left\{x_{z, s}\right\}_{z \in \mathcal{Z}, s \in \mathcal{S}},\left\{\mathcal{I}_{i, s}, \mathcal{W}_{i, s}\right\}_{i \in \mathcal{N}, s \in \mathcal{S}}\right)=\sum_{z \in \mathcal{Z}} \sum_{i \in \mathcal{B}(z)} \sum_{s \in \mathcal{S}} g_{z, i, s}\left(x_{z, s}, \mathcal{I}_{i, s}, \mathcal{W}_{i, s}\right)
$$

Constraints (2)-(3), (5)-(6) and (16) can be rewritten as,

$$
A_{i, s}\left[\mathcal{P}_{i, s},\left\{\mathcal{P}_{j, s}\right\}_{(i, j) \in \mathcal{L}}, \mathcal{I}_{i, s}, \mathcal{W}_{i, s}, x_{z, s}\right]^{T} \leq a_{i, s} ; \forall z \in \mathcal{Z}, i \in \mathcal{B}(z), s \in \mathcal{S}
$$

where $A_{i, s}$ and $a_{i, s}$ are the corresponding coefficient matrixes. It is obvious that adjacent nodes $(i, j) \in \mathcal{L}$ are coupled through $\mathcal{P}_{i, s}$ and $\mathcal{P}_{j, s}$ in (26), which results in a coupling between adjacent zones $\hat{z}_{i}$ and $\hat{z}_{j}$.

Constraints (4), (8)-(11), (17)-(18), and (23) are separable for each zone and are rewritten as,

$$
B_{i, s}\left[\mathcal{P}_{i, s}, \mathcal{I}_{i, s}, \mathcal{W}_{i, s}, x_{z, s}\right]^{T} \leq b_{i, s} ; \forall z \in \mathcal{Z}, i \in \mathcal{B}(z), s \in \mathcal{S}
$$

where $B_{i, s}$ and $b_{i, s}$ are the corresponding coefficient matrixes.

Constraints (12)-(13), (15)-(16) and (24) can be rewritten as,

$$
C\left[\left\{\mathcal{W}_{i, s}\right\}_{i \in \mathcal{N}, s \in \mathcal{S}},\left\{x_{z, s}\right\}_{z \in \mathcal{Z}, s \in \mathcal{S}}\right]^{T} \leq c
$$

where $C$ and $c$ are the corresponding coefficient matrixes.

Then, the MILP model is expressed by the compact form in (25)-(28). To use the ADMM to distribute the MILP model, four sets of auxiliary variables $\left(\tilde{\mathcal{P}}_{i, s}, \hat{\mathcal{P}}_{i, s}^{j}, \tilde{\mathcal{W}}_{i, s}, \tilde{x}_{z, s}\right)$ and equality constraints are introduced as shown in (29e). By replacing the original variables in (25)-(27) with the auxiliary variables, the MILP model is rewritten as,

$$
\min f\left(\tilde{x}_{z, s}, \mathcal{I}_{i, s}, \tilde{\mathcal{W}}_{i, s}\right)
$$

s.t.

$$
\begin{aligned}
& A_{i, s}\left[\tilde{\mathcal{P}}_{i, s},\left\{\hat{\mathcal{P}}_{i, s}^{j}\right\}_{(i, j) \in \mathcal{L}}, \mathcal{I}_{i, s}, \tilde{\mathcal{W}}_{i, s}, \tilde{x}_{z, s}\right]^{T} \leq a_{i, s} ; \quad \forall z \in \mathcal{Z}, i \in \mathcal{B}(z), s \in \mathcal{S} \\
& B_{i, s}\left[\tilde{\mathcal{P}}_{i, s}, \mathcal{I}_{i, s}, \tilde{\mathcal{W}}_{i, s}, \tilde{x}_{z, s}\right] \leq b_{i, s} ; \forall z \in \mathcal{Z}, i \in \mathcal{B}(z), s \in \mathcal{S} \\
& C\left[\left\{\mathcal{W}_{i, s}\right\}_{i \in \mathcal{N}, s \in \mathcal{S}},\left\{x_{z, s}\right\}_{z \in \mathcal{Z}, s \in \mathcal{S}}\right]^{T} \leq c ; \\
& \left\{\begin{array}{l}
\mathcal{P}_{i, s}=\tilde{\mathcal{P}}_{i, s}, \mathcal{P}_{j, s}=\hat{\mathcal{P}}_{i, s}^{j} ; \quad \forall i \in \mathcal{N},(i, j) \in \mathcal{L}, s \in \mathcal{S} \\
\mathcal{W}_{i, s}=\tilde{\mathcal{W}}_{i, s} ; x_{z, s}=\tilde{x}_{z, s} ; \quad \forall i \in \mathcal{N}, z \in \mathcal{Z}, s \in \mathcal{S} \\
\tilde{\mathcal{P}}_{i, s}, \hat{\mathcal{P}}_{i, s}^{j}, \tilde{\mathcal{W}}_{i, s}, \tilde{x}_{z, s} \in \mathbb{R}
\end{array}\right.
\end{aligned}
$$


Note that, those variables with the subscript of $i$ exclusively belong to node $i$. Therefore, (29b) and (29c) can be decomposed among each zone.

\subsection{ADMM -based Algorithm}

\subsubsection{Augmented Lagrangian}

For brevity, define sets $\mathcal{X}_{1}, \mathcal{X}_{2}$ and $\mathcal{V}$ as below,

$$
\left\{\begin{array}{l}
\mathcal{X}_{1}:=\left\{\left\{\tilde{\mathcal{P}}_{i, s}, \hat{\mathcal{P}}_{i, s}^{j}, \mathcal{I}_{i, s}, \tilde{\mathcal{W}}_{i, s}, \tilde{x}_{z, s}\right\}_{i \in \mathcal{N},(i, j) \in \mathcal{L}, s \in \mathcal{S}, z \in \mathcal{Z}} \mid(29 \mathrm{~b}),(29 \mathrm{c})\right\} \\
\mathcal{X}_{2}:=\left\{\left\{\mathcal{P}_{i, s}, \mathcal{W}_{i, s}, x_{z, s}\right\}_{i \in \mathcal{N}, s \in \mathcal{S}, z \in \mathcal{Z}} \mid(29 \mathrm{~d})\right\} ; \\
\mathcal{V}:=\left\{\left\{\mathcal{A}_{i, s}, \mathcal{B}_{i, s}, \mathcal{C}_{i, s}\right\}_{i \in \mathcal{N}, s \in \mathcal{S}},\left\{\mathcal{D}_{i, s}^{j}\right\}_{(i, j) \in \mathcal{L}, s \in \mathcal{S}}\right\} ;
\end{array}\right.
$$

where $\mathcal{X}_{1}$ and $\mathcal{X}_{2}$ are the sets of primal variables and $\mathcal{V}$ is the set of dual variables. Then, by adding equality constraint (29e) into the objective function (29a) through the dual variables in $\mathcal{V}$, the augmented Lagrangian of the MILP problem is formulated as,

$$
\begin{array}{r}
\min _{\mathcal{X}_{1}, \mathcal{X}_{2}, \mathcal{v}} f\left(\tilde{x}_{z, s}, \mathcal{I}_{i, s}, \tilde{\mathcal{W}}_{i, s}\right)+\sum_{s \in \mathcal{S}} \sum_{z \in \mathcal{Z}} \frac{\rho_{z}}{2}\left\{\left(\left\|x_{z, s}-\tilde{x}_{z, s}+\mathcal{A}_{z, s}\right\|^{2}-\left\|\mathcal{A}_{z, s}\right\|^{2}\right)\right. \\
+\sum_{i \in \mathcal{N}(\mathcal{Z})}\left(\left\|\mathcal{P}_{i, s}-\tilde{\mathcal{P}}_{i, s}+\mathcal{B}_{i, s}\right\|^{2}-\left\|\mathcal{B}_{i, s}\right\|^{2}+\left\|\mathcal{W}_{i, s}-\tilde{\mathcal{W}}_{i, s}+\mathcal{C}_{i, s}\right\|^{2}-\left\|\mathcal{C}_{i, s}\right\|^{2}\right) \\
\left.+\sum_{i \in \mathcal{N}(\mathcal{Z}) \cap(i, j) \in \mathcal{L}}\left(\left\|\mathcal{P}_{j, s}-\hat{\mathcal{P}}_{i, s}^{j}+\mathcal{D}_{i, s}^{j}\right\|^{2}-\left\|\mathcal{D}_{i, s}^{j}\right\|^{2}\right)\right\}
\end{array}
$$

Then, the ADMM-based algorithm is used to solve the augmented Lagrangian in an iterative manner.

\subsubsection{Sub-problem I}

Sub-problem I is a quadratic programming (QP) problem given by (27). At the $k$-th iteration, variables in $\mathcal{X}_{1}$ are optimized and other variables are fixed as the values obtained in the (k-1)-th iteration, i.e., $\mathcal{X}_{2}^{k-1}$ and $\mathcal{V}^{k-1}$.

$$
\begin{aligned}
& \min _{\mathcal{X}_{1}} f\left(\tilde{x}_{z, s}, \mathcal{I}_{i, s}, \tilde{\mathcal{W}}_{i, s}\right)+ \sum_{s \in \mathcal{S}} \sum_{z \in \mathcal{Z}} \frac{\rho_{z}}{2}\left\{\left\|x_{z, s}^{k-1}-\tilde{x}_{z, s}+\mathcal{A}_{z, s}^{k-1}\right\|^{2}\right. \\
&+\sum_{i \in \mathcal{N}(\mathcal{Z})}\left(\left\|\mathcal{P}_{i, s}^{k-1}-\tilde{\mathcal{P}}_{i, s}+\mathcal{B}_{i, s}^{k-1}\right\|^{2}+\left\|\mathcal{W}_{i, s}^{k-1}-\tilde{\mathcal{W}}_{i, s}+\mathcal{C}_{i, s}^{k-1}\right\|^{2}\right) \\
&\left.+\sum_{i \in \mathcal{N}(\mathcal{Z}) \cap(i, j) \in \mathcal{L}}\left(\left\|\mathcal{P}_{j, s}^{k-1}-\hat{\mathcal{P}}_{i, s}^{j}+\mathcal{D}_{i, s}^{j, k-1}\right\|^{2}\right)\right\}
\end{aligned}
$$

The objective function in (30) and constraints (29b) and (29c) are separable for each zone. Thus, variables in $\mathcal{X}_{1}$ can be optimized separately in parallel. In sub-problem I, each zone controller solves its sub-problem locally.

\subsubsection{Sub-problem II}

Sub-problem II is a mixed-integer quadratic programming (MIQP) problem shown in (31). At the $k$-th iteration, variables in $\mathcal{X}_{2}$ are optimized with obtained $\mathcal{X}_{1}{ }^{k}$ and $\mathcal{V}^{k-1}$.

$$
\begin{aligned}
& \min _{\mathcal{X}_{2}} \sum_{s \in \mathcal{S}} \sum_{z \in \mathcal{Z}} \frac{\rho_{z}}{2}\left\{\left\|x_{z, s}-\tilde{x}_{z, s}^{k}+\mathcal{A}_{z, s}^{k-1}\right\|^{2}+\sum_{i \in \mathcal{N}(\mathcal{Z})}\left(\left\|\mathcal{P}_{i, s}-\tilde{\mathcal{P}}_{i, s}^{k}+\mathcal{B}_{i, s}^{k-1}\right\|^{2}\right.\right. \\
&\left.\left.+\left\|\mathcal{W}_{i, s}-\tilde{\mathcal{W}}_{i, s}^{k}+\mathcal{C}_{i, s}^{k-1}\right\|^{2}\right)+\sum_{j \in \mathcal{N}(\mathcal{Z}) \cap(i, j) \in \mathcal{L}}\left(\left\|\mathcal{P}_{j, s}-\hat{\mathcal{P}}_{i, s}^{j, k}+\mathcal{D}_{i, s}^{j, k-1}\right\|^{2}\right)\right\}
\end{aligned}
$$


In (31), $\mathcal{W}_{i, s}$ and $x_{z, s}$ are coupled in $\mathcal{X}_{2}$, as shown in (29d). Therefore, variables in $\mathcal{W}_{i, s}$ and $x_{z, s}$ are optimized in a centralized way by the central controller. Note that, variables in $\mathcal{P}_{i, s}$ are free variables and the associated terms in the objective function are separable for each zone. Therefore, variables in $\mathcal{P}_{i, s}$ can be optimized separately by local zone controllers.

\subsubsection{Dual variables update and stopping criterion}

After solving sub-problems I and II, dual variables are updated using (32) at the $k$-th iteration. In the ADMM-based algorithm, primal and dual variables are updated iteratively. The iteration process stops till aggregated primal residuals $r_{z}^{k}$ and dual residuals $d_{z}^{k}$ are lower than specified thresholds $\varepsilon_{1}, \varepsilon_{2}$, as shown in (33) and (34).

$$
\begin{aligned}
& \begin{cases}\mathcal{A}_{z, s}^{k}=\mathcal{A}_{z, s}^{k-1}+\left(x_{z, s}^{k}-\tilde{\mathcal{X}}_{z, s}^{k}\right) ; & \forall z \in \mathcal{Z}, s \in \mathcal{S} \\
\mathcal{B}_{i, s}^{k}=\mathcal{B}_{i, s}^{k-1}+\left(\mathcal{P}_{i, s}^{k}-\tilde{\mathcal{P}}_{i, s}^{k}\right) ; & \forall i \in \mathcal{N}, s \in \mathcal{S} \\
\mathcal{C}_{i, s}^{k}=\mathcal{C}_{i, s}^{k-1}+\left(\mathcal{W}_{i, s}^{k}-\tilde{\mathcal{W}}_{i, s}^{k}\right) ; & \forall i \in \mathcal{N}, s \in \mathcal{S} \\
\mathcal{D}_{i, s}^{j, k}=\mathcal{D}_{i, s}^{j, k-1}+\left(\mathcal{P}_{j, s}^{k}-\hat{\mathcal{P}}_{i, s}^{j, k}\right) ; \forall(i, j) \in \mathcal{L}, s \in \mathcal{S}\end{cases} \\
& \left\{\begin{array}{l}
r_{z}^{k}=\sum_{s \in \mathcal{S}}\left\{\left\|x_{z, s}^{k}-\tilde{x}_{z, s}^{k}\right\|^{2}+\sum_{i \in \mathcal{N}(Z)}\left(\left\|\mathcal{P}_{i, s}^{k}-\tilde{\mathcal{P}}_{i, s}^{k}\right\|^{2}+\left\|\mathcal{W}_{i, s}^{k}-\tilde{\mathcal{W}}_{i, s}^{k}\right\|^{2}\right)+\sum_{i \in \mathcal{N}(z) \cap(i, j) \in \mathcal{L}}\left(\left\|\mathcal{P}_{j, s}^{k}-\hat{\mathcal{P}}_{i, s}^{j, k}\right\|^{2}\right)\right\} ; \forall z \in \mathcal{Z} \\
d_{z}^{k}=\sum_{s \in \mathcal{S}}\left\{\left\|x_{z, s}^{k}-x_{z, s}^{k-1}\right\|^{2} \sum_{i \in \mathcal{N}(z)}\left(\left\|\tilde{\mathcal{P}}_{i, s}^{k}-\tilde{\mathcal{P}}_{i, s}^{k-1}\right\|^{2}+\left\|\mathcal{W}_{i, s}^{k}-\mathcal{W}_{i, s}^{k-1}\right\|^{2}\right)+\sum_{i \in \mathcal{N}(z) \cap(i, j) \in \mathcal{L}}\left(\left\|\hat{\mathcal{P}}_{i, s}^{j, k}-\hat{\mathcal{P}}_{i, s}^{j, k-1}\right\|^{2}\right)\right\} ; \forall z \in \mathcal{Z}
\end{array}\right. \\
& \sum_{z \in \mathcal{Z}} r_{z}^{k} \leq \varepsilon_{1} \& \sum_{z \in \mathcal{Z}} d_{z}^{k} \leq \varepsilon_{2}
\end{aligned}
$$

\subsubsection{Penalty parameter tuning}

A distributed penalty parameter tuning technique in [30] is used to improve the convergence performance of the proposed ADMM-based algorithm. The penalty parameter $\rho_{z}$ for each zone is updated according to primal and dual residuals of each zone, as shown in (35). The penalty parameter is updated in such a fashion in order to balance convergence of primal and dual residuals. As discussed in [30], a larger $\rho^{k}$ places a larger penalty on violations of primal feasibility and decrease primal residuals more effectively. In contrast, a smaller $\rho^{k}$ produces smaller dual residuals and more accurate solutions.

$$
\rho_{z}^{k+1}= \begin{cases}\rho_{z}^{k} / 1+\tau ; & \text { if }\left\|r_{z}^{k}\right\| \leq \mu\left\|d_{z}^{k}\right\| \\ (1+\tau) \rho_{z}^{k} ; & \text { if }\left\|d_{z}^{k}\right\| \leq \mu\left\|r_{z}^{k}\right\| \\ \rho_{z}^{k} ; & \text { otherwise }\end{cases}
$$

\subsection{Information Exchanges in the Hierarchal Framework}

\subsubsection{Information exchanges in sub-problem I}

In sub-problem I, zone controllers collect $\mathcal{X}_{2}^{*}$, i.e., $x_{z, s}^{*}, \mathcal{W}_{i, s}^{*}$ and $\mathcal{P}_{i, s}^{*}$, from the central controller and solve local subproblems in parallel to optimize $\mathcal{X}_{1}$, as shown in Fig. 2a. 


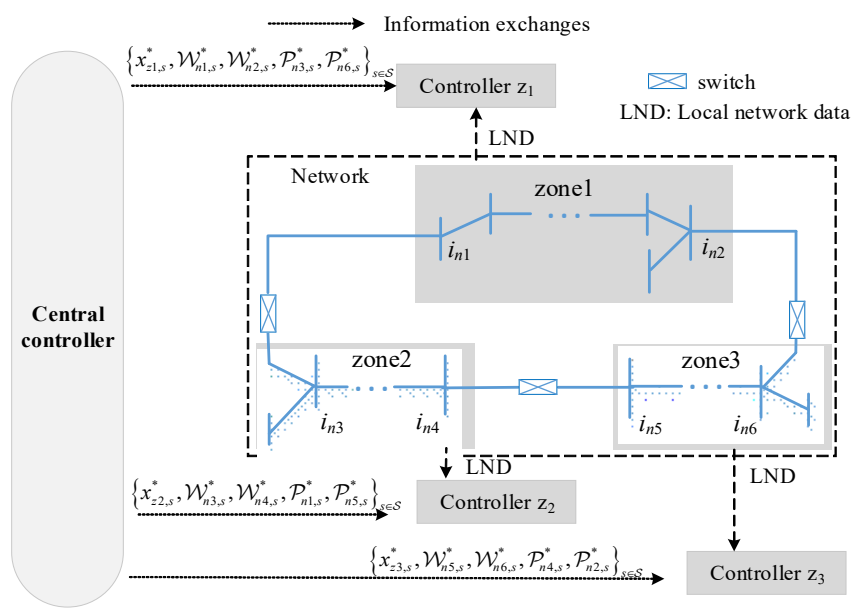

Fig. 2a. Information exchanges in sub-problem I.

\subsubsection{Information Exchanges in sub-problem II}

In sub-problem II, the central controller solves a global problem and zone controllers solve local sub-problems to optimize $\mathcal{X}_{2}$. As shown in Fig. $2 b$, to optimize $\mathcal{X}_{2}$, the central controller collects a part of $\mathcal{X}_{1}^{*}$, i.e., $\tilde{x}_{z, s}^{*}, \tilde{\mathcal{W}}_{i, s}^{*}$ and $\hat{\mathcal{P}}_{i, s}^{*, j}$, from zone controllers and zone controllers collect $\hat{\mathcal{P}}_{i, s}^{*, j}$ from the central controller.

The information exchanges between zone controllers and the central controller are limited because only information of status of zones and boundary nodes is exchanged, which enhances the privacy information protection.

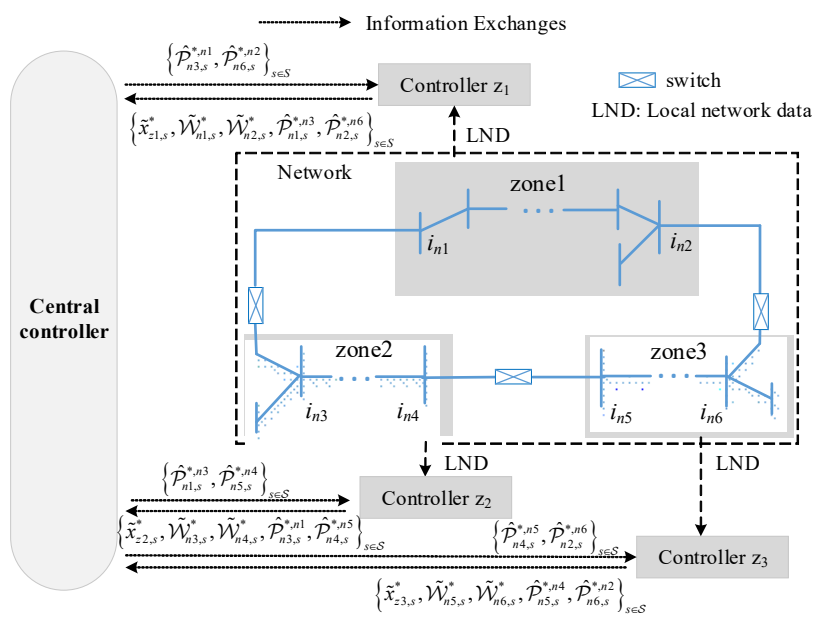

Fig. 2b. Information exchanges in sub-problem II.

\section{Case Studies}

Three case studies were conducted with the 44-node test system, modified IEEE 123-node system and Brazil distribution system to validate the effectiveness of the proposed hierarchical service restoration scheme. Simulations were carried out using the GAMS with the CPLEX solver. In the proposed ADMM-based algorithm, parameters $c_{i}^{\mathrm{G}}$ is 0.1 m.u. (monetary unit)/MW for DG units and 0.2 m.u./MW for main sources, $c_{i}^{j, \text { lss }}$ is 0.1 m.u./MW, $c_{i}^{\mathrm{r}}$ is 5 m.u./MW and $c_{i}^{j, \mathrm{~s}}$ is 0.1 m.u.. The threshold $\varepsilon_{1}$ is set as 0.001 in cases 1 and 2 and 0.01 in case 3 , and $\varepsilon_{2}$ is set as 0.01 in all cases. The number of discrete segments $|\mathcal{P}|$ and the maximum 
number of switching operations $s_{\max }$ are set as 30 and 8, respectively. The lower bound of voltage magnitudes is set as 0.95 p.u..

\subsection{Case 1: 44-node test system}

The 44-node test system in Fig. 3 consists of three feeders and nine zones with two DG units connected at zone 3 (DG1) and zone 9 (DG2). DG1 and DG2 operate with the unity power factor and their capacities are $100 \mathrm{~kW}$. Closed switches and open switches are represented by black and white colored boxes, respectively. The detailed data of loads and circuits can be found in [18]. Three scenarios were considered to validate the effectiveness of the proposed hierarchical service restoration scheme.

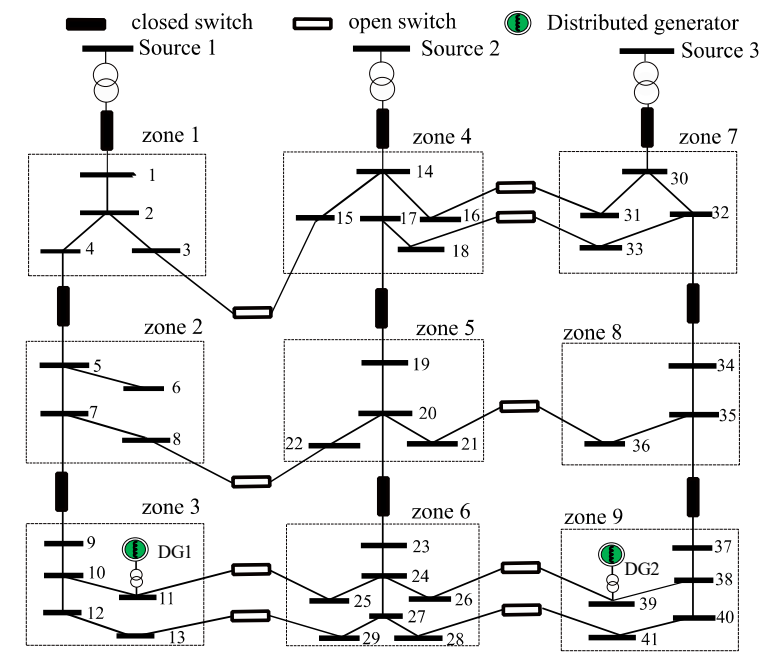

Fig. 3. Initial topology of the 44 -node test system

\subsubsection{Scenario 1: with DGs and load shedding}

It is assumed that a permanent fault occurs in zone 1. The results of service restoration are summarized in Table 1. The minimum voltage magnitude during service restoration, switching sequence and objective function values are given. As shown in Table 1, the switches between source node 1 and node 1 and between nodes 4 and 5 are opened to isolate the faulted zone 1 at steps $\mathrm{s}_{1}$ and $\mathrm{s}_{2}$, respectively. Then, the switch between nodes 8 and 22 is closed to resupply outage zones 2 and 3 at step $\mathrm{s}_{3}$. In this scenario, operational constraints are not violated and two DG units operate at their maximum output after service restoration because they have lower energy costs than the main sources.

\subsubsection{Scenario 2: without DG1 but with load shedding}

In this scenario, the DG1 is disconnected and the same fault occurs in zone 1. As shown in Table 1, at step s3, outage zones 2 and 3 are supplied by closing the switch between nodes 11 and 25. To ensure that voltage magnitudes are maintained within limits at this step, $23.50 \mathrm{~kW}$ and $4.70 \mathrm{kVar}$ loads at node 5 , and $23.5 \mathrm{~kW}$ and $4.70 \mathrm{kVar}$ loads at node 8 are shed. The switch between nodes 11 and 25 is closed instead of the switch between nodes 8 and 22 at step s 3 because of the limited current capacity of the switch between nodes 8 and 22. Since the operation of DG1 is disregarded and more energy must be provided from main source 2 to supply loads in zone 3, closing the switch between nodes 8 and 22 results in congestion and a higher cost of load shedding. Finally, outage zones 2 and 3 are supplied separately by opening the switch between nodes 7 and 9 and closing the switch between nodes 8 and 22. During service restoration, the minimal voltage magnitude is 0.95 p.u. at nodes 5 and 6 at step s. Compared with scenario 1 , scenario 2 requires more switching operations and has a higher total cost. It is obvious that DG units can improve the restoration ability of the ADN.

\subsubsection{Scenario 3: with DGs but without load-shedding}

In this scenario, the same fault occurs in zone 1 but load shedding is not allowed. The switching operations are given in Table I. Different from scenario 2, the switch between nodes 7 and 9 is opened firstly to separate outages zones 2 and 3 before 
restoring them. Then, outage zone 2 is restored at step $s_{4}$ by closing the switch between nodes 8 and 22 and zone 3 is restored at step $\mathrm{s}_{5}$ by closing the switch between nodes 11 and 25 . In this scenario, operational constraints are not violated. Compared with scenario 2, scenario 3 has a higher total cost because zones 2 and 3 can be restored only when zones are restored completely. Therefore, the appropriate load shedding can facilitate service restoration.

Table 1

Results of Service Restoration in Case 1

\begin{tabular}{|c|c|c|c|c|}
\hline \multicolumn{2}{|c|}{ Scenarios } & no. 1 & no. 2 & no. 3 \\
\hline \multirow{4}{*}{ Switching sequence } & $\mathrm{s}_{1}$ & $\mathrm{~s} 1-1 \uparrow^{1}$ & $\mathrm{~s} 1-1 \uparrow$ & $\mathrm{s} 1-1 \uparrow$ \\
\hline & $\mathrm{s}_{2}$ & $4-5 \uparrow$ & $4-5 \uparrow$ & $4-5 \uparrow$ \\
\hline & $\mathrm{S}_{4}$ & - & $7-9 \uparrow$ & $8-22 \downarrow$ \\
\hline & $\mathrm{s}_{5}$ & - & $8-22 \downarrow$ & $11-25 \downarrow$ \\
\hline \multicolumn{2}{|c|}{ O.F. $^{3}$} & 11.799 & 13.444 & 14.988 \\
\hline \multicolumn{2}{|c|}{ Num. $^{4}$} & 898 & 1771 & 1117 \\
\hline
\end{tabular}

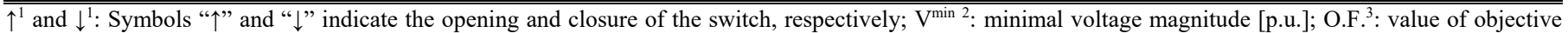
function [m.u.]; Num. ${ }^{4}$ : number of iterations of the ADMM-based algorithm.

\subsection{Case 2: IEEE 123-node system}

The modified IEEE 123-node system in Fig. 5 is used to further demonstrate the proposed hierarchical service restoration scheme. The system has two feeders and seven zones with three DG units connected at zones 3 (DG1), 5 (DG2) and 7 (DG3). DG units operate with the unity power factor and their capacities are $100 \mathrm{~kW}, 15 \mathrm{~kW}$ and $80 \mathrm{~kW}$, respectively. Detailed parameters of circuits and loads can be found in [31].

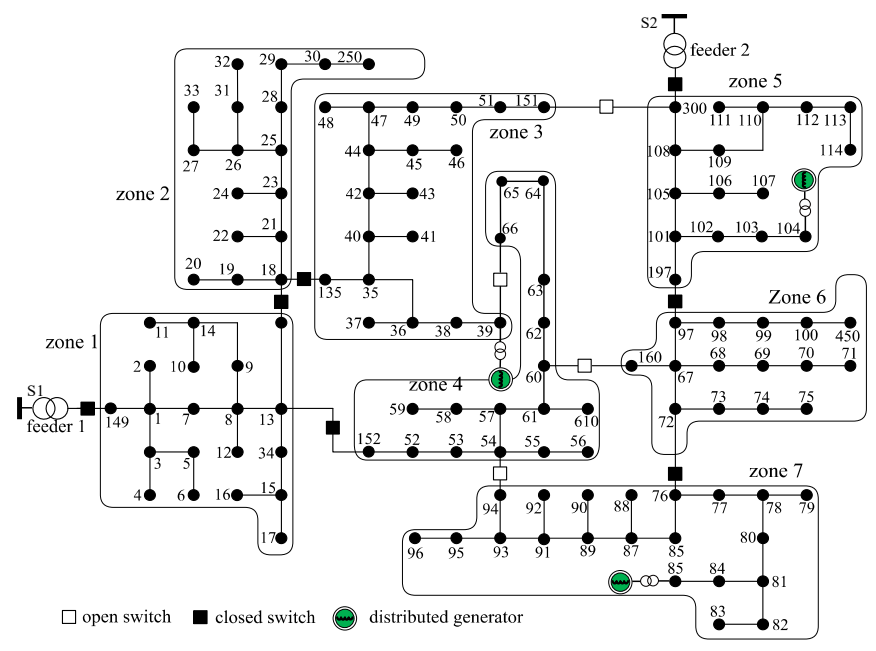

Fig. 5. Initial topology of the modified IEEE 123-node system

In this case study, all possible faulted zones were tested. The results of service restoration are summarized in Table 2. It can be seen that the proposed hierarchical scheme can restore the system while maintaining the faulted zone isolated in all tests. Moreover, the proposed scheme guarantees that operational constraints are not violated and the energized portion of the system has a radial topology at each step.

As shown in Table 2, if the fault occurs in zone 3, zone 4 or zone 7, the service restoration process requires only one switch operation because these zones locate at the end of feeders and the isolation of these zones will not affect healthy zones. When the fault occurs in other zones, i.e., zones 1,2,5 and 6, additional switching operations are required to restore outage zones. In addition, the numbers of iterations of the ADMM-based algorithm are listed in Table 2. 
Table 2

Results of Service Restoration in Case 2

\begin{tabular}{|c|c|c|c|c|c|c|c|c|}
\hline & & \multicolumn{7}{|c|}{ "Faulty zone } \\
\hline & & 1 & 2 & 3 & 4 & 5 & 6 & 7 \\
\hline \multirow{3}{*}{$\mathrm{sw}^{1}$} & $\mathrm{~s}_{1}$ & $\mathrm{~s} 1-149 \uparrow$ & $13-18 \uparrow$ & $18-135 \uparrow$ & $13-152 \uparrow$ & $\mathrm{s} 2-300 \uparrow$ & $97-197 \uparrow$ & $72-76 \uparrow$ \\
\hline & $\mathrm{s}_{3}$ & $13-152 \uparrow$ & $151-300 \downarrow$ & - & - & $\begin{array}{l}54-94 \downarrow \\
\end{array}$ & 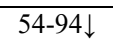 & - \\
\hline & $\mathrm{s}_{4}$ & $151-300 \downarrow$ & - & - & - & $72-76 \uparrow$ & - & - \\
\hline \multicolumn{2}{|c|}{ Num. } & 615 & 607 & 445 & 243 & 723 & 445 & 362 \\
\hline
\end{tabular}

$\mathrm{sw}^{1}$ : Switching sequence

\subsection{Case 3: Brazil 948-node Distribution System}

The modified Brazil 948-node distribution system shown in Fig. 6 is used to demonstrate the scalability of the proposed hierarchical scheme. The system consists of 948 nodes, 9 DGs, 85 loads zones and 95 switches. The detailed parameters of transmission lines and loads are available online [32]. The capacity of DGs units in zones 51, 55, 40, 47, 85, 83 is $50 \mathrm{~kW}$ and of the capacity of other DGs is $20 \mathrm{~kW}$.

Suppose that a permanent fault occurs in zone 48 at feeder 2. After implementing the proposed ADMM-based algorithm, the hierarchical scheme isolates the faulty zone at the first three restoration steps, i.e., the switch between zones 25 and 48 is opened at $s_{1}$ to isolate the faulty zone from the upstream substation. The switches between zones 48 and 50 and between zones 48 and 49 are opened at $s_{2}$ and $s_{3}$, respectively, to separate the faulty zone from the downstream outage zones 49-55. Then, at step $s_{4}$, outage zones 49, 51-55 are supplied by closing the switch between zones 32 and 49. Finally, feeder 1 supplies the outage zone 50 by closing the tie-switch between zones 50 and 85 at step $s_{6}$. The system after reconfiguration has a radial topology with the minimum voltage magnitude of 0.975 p.u. at node 925 in zone 38. Moreover, no load shedding is needed and all DG units use their maximum capacity. In this case, the proposed ADMM-based algorithm needs 1523 iterations to converge.

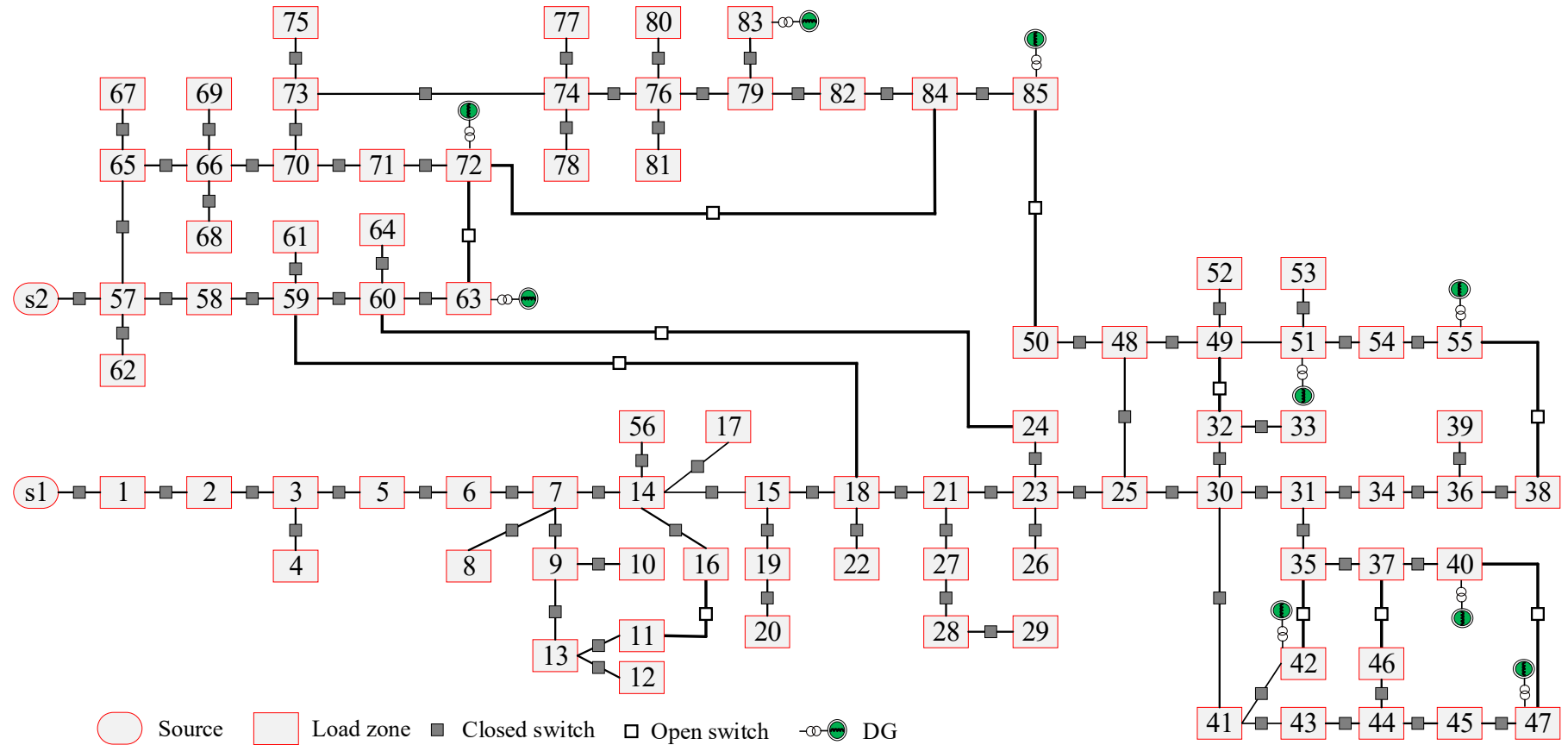

Fig. 6. Initial topology of the modified Brazil 948-node distribution system 
5.4 Sensitivity Analysis to the Penalty Parameter $\rho$

Case 1 is used to conduct the sensitivity analysis by implementing the proposed ADMM-based algorithm with different penalty parameters between 50 and 400 . Table 3 shows the numbers of iterations in three scenarios of case 1 with and without the penalty parameter tuning technique. It can be seen that, within a certain parameter range, the algorithm has better convergence performance as $\rho^{0}$ increases. However, it may have slow convergence if $\rho^{0}$ is too large, e.g., $\rho^{0}=400$. Moreover, Table 3 shows that the algorithm with the penalty parameter tuning technique has smaller average numbers of iterations. In most of the scenarios, the convergence process can be accelerated by using the varying penalty parameter. Fig. 4 shows the convergence processes of primal and dual residuals with the penalty parameter tuning technique and $\rho^{0}=200$, which demonstrates the feasibility and effectiveness of the proposed ADMM-based algorithm.

Table 3

Numbers of Iterations in Three Scenarios in Case 1 without and with the Penalty Parameter Tuning Technique

\begin{tabular}{c|cccc|cccc}
\hline \hline Num. & \multicolumn{4}{|c|}{ without $\rho$ tuning technique } & \multicolumn{3}{c}{ with $\rho$ tuning technique } \\
\hline$\rho^{0}$ & scenario 1 & scenario 2 & scenario 3 & average & scenario 1 & scenario 2 & scenario 3 & average \\
\hline 50 & 1520 & 1798 & 1187 & 1501.7 & 1168 & 1571 & 1552 \\
\hline 100 & 1127 & 1771 & 1208 & 1368.7 & 1060 & 1067 & 1325 & 1150.7 \\
\hline 200 & 806 & 1406 & 1186 & 1132.6 & 808 & 1165 & 1204 & 1059.0 \\
\hline 300 & 991 & 1840 & 1334 & 1388.3 & 1100 & 886 & 1356 & 1114.0 \\
\hline 400 & 930 & 1947 & 1295 & 1424.0 & 792 & 983 & 1087.3 \\
\hline \hline
\end{tabular}
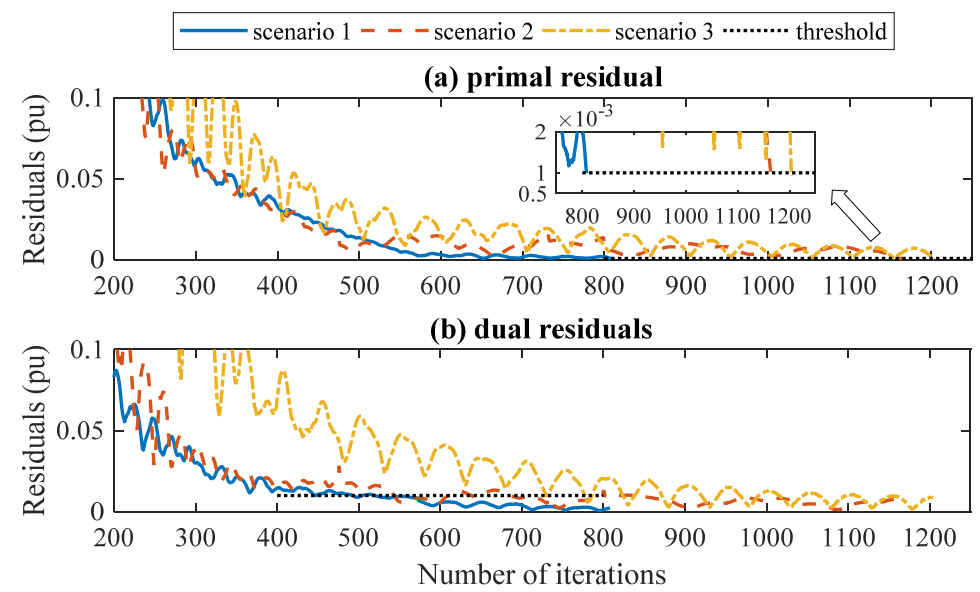

Fig. 7. Convergence of the ADMM-based algorithm with the penalty tuning technique $\left(\rho^{0}=200\right)$

\subsection{Discussions on Quality of Solutions and Computation Efficiency}

Comparisons between solutions obtained with the proposed hierarchical and centralized schemes in case 1 are given in Table 4. The centralized solution is obtained by solving the MILP model in Section II using the CPLEX solver with a maximum optimality gap of $1 \%$. Table 4 lists objective function values and power generation of the two schemes in case 1 . It can be seen that power generation in the two schemes are almost the same and the relative error of objective function values is lower than $0.1 \%$, which demonstrates that the proposed hierarchical scheme can reproduce the centralized solution.

Table 5 summarizes computation complexity of the centralized and hierarchical schemes in case 3. Compared with the centralized scheme, the average number of continuous variables of each controller in each calculation is reduced by $99.4 \%$ in the hierarchical scheme. Moreover, zone controllers optimize continuous variables only and the central controller optimizes binary variables only. Therefore, the computational complexity of the service restoration problem is reduced substantially by using the proposed hierarchical scheme. 
The computation time of all cases with the centralized and hierarchical schemes is compared in Table 6. The hierarchical scheme can obtain solutions within $120 \mathrm{~s}$ in all cases, which complies with requirements of real applications. As for 44-node and 123-node systems, the centralized scheme needs less computation time than the hierarchical scheme. This is because the centralized algorithm is capable of solving small-scale problems whereas the ADMM-based algorithm needs iterations to obtain solutions. However, for the large-scale Brazil 948-node system, the computation time of the ADMM-based algorithm is $115 \mathrm{~s}$ that is $96.15 \%$ lower than the one with the centralized algorithm. The centralized algorithm suffers from a heavy computation burden because the computation complexity grows exponentially as the number of binary variables increase. In contrast, the ADMM-based algorithm can decompose the large-scale problem into smaller-scale problems and solve them efficiently.

In summary, the hierarchical scheme can obtain optimal solutions with less computation complexity. Especially for largescale systems, the computation time decreases substantially by using the hierarchical scheme.

Table 4

The Comparison of Solutions in Two Schemes in Case 1

\begin{tabular}{|c|c|c|c|}
\hline Scenario & Values & Centralized scheme & Hierarchical scheme \\
\hline \multirow{5}{*}{ no. 1} & O.F. & 11.799 & 11.799 \\
\hline & APSN $2^{1}$ & 0.715 & 0.715 \\
\hline & APSN 3 & 0.612 & 0.612 \\
\hline & RPSN $2^{2}$ & 0.175 & 0.175 \\
\hline & RPSN 3 & 0.152 & 0.152 \\
\hline \multirow{5}{*}{ no. 2} & O.F. & 13.444 & 13.455 \\
\hline & APSN 2 & 0.820 & 0.820 \\
\hline & APSN 3 & 0.612 & 0.612 \\
\hline & RPSN 2 & 0.180 & 0.180 \\
\hline & RPSN 3 & 0.152 & 0.152 \\
\hline \multirow{5}{*}{ no. 3} & O.F. & 14.988 & 14.988 \\
\hline & APSN 2 & 0.820 & 0.820 \\
\hline & APSN 3 & 0.612 & 0.612 \\
\hline & RPSN 2 & 0.180 & 0.180 \\
\hline & RPSN 3 & 0.152 & 0.152 \\
\hline
\end{tabular}

${ }^{1}$ APSN $i$ : Active power source at source node $i$ (MW)

${ }^{2}$ RPSN $i$ : Reactive power source at source node $i$ (MVar)

Table 5

Comparisons of Computational Complexity between Two Schemes in Case 3

\begin{tabular}{|c|c|c|c|}
\hline \multirow{2}{*}{ The number of variables } & Centralized scheme & \multicolumn{2}{|c|}{ Hierarchical scheme } \\
\hline & Central controller & Zone controller & Central controller \\
\hline Continuous variables & 481216 & 2860 & 0 \\
\hline Integer variables & 2476 & 0 & 2476 \\
\hline
\end{tabular}

Table 6

Computation Time of Two Schemes in Three Cases

\begin{tabular}{|c|c|c|c|c|c|c|c|c|c|c|c|}
\hline $\begin{array}{l}\text { Computation } \\
\text { time (s) }\end{array}$ & \multicolumn{3}{|c|}{ 44-ndoe system } & \multicolumn{7}{|c|}{ 123-node system } & \multirow{2}{*}{$\begin{array}{c}\text { real Brazil } \\
\text { system }\end{array}$} \\
\hline Scenarios & no. 1 & no. 2 & no. 3 & no.1 & no. 2 & no. 3 & no. 4 & no. 5 & no.6 & no. 7 & \\
\hline Centralized & 2.3 & 5.5 & 4.8 & 8.9 & 9.6 & 8.4 & 8.9 & 11.2 & 10.4 & 10.2 & 2993.3 \\
\hline Hierarchical & 22.1 & 41.9 & 54.2 & 48.0 & 44.9 & 29.6 & 17.6 & 73.8 & 34.0 & 21.8 & 115.1 \\
\hline
\end{tabular}




\section{Conclusions}

This paper proposes a hierarchical service restoration scheme for ADNs based on the ADMM. The optimal service restoration problem is formulated as a MINLP model. The MINLP model is transformed into a MILP model using linearization techniques, which is decomposed and solved by the ADMM-based algorithm. Through decomposition, the service restoration problem is solved in a hierarchical framework, in which each zone controller solves its local sub-problem with information from the central controller and the central controller solves a global coordination problem with information from each zone controller. The simulation results demonstrate that the proposed hierarchical service restoration scheme can restore the ADN with a radial topology and guarantee that operation constraints are not violated. Compared with the centralized scheme, the average number of continuous variables of each controller in each calculation is reduced by $99.4 \%$ in the hierarchical scheme. Moreover, zone controllers optimize continuous variables only and the central controller optimizes binary variables only. Moreover, the solution obtained with the proposed hierarchical scheme is the same as the one obtained with the centralized scheme. Therefore, the proposed hierarchical scheme can obtain the optimal solutions with less computational complexity. In addition, as for large-scale ADNs, the proposed hierarchical scheme has a less computation burden. For communication architecture, information exchanges in the proposed hierarchical scheme are limited because only variables of boundary nodes and variables associated with the status of zones are exchanged, which enhances the privacy information protection as compared with the centralized scheme.

\section{References}

[1] D. Manz, R. Walling, N. Miller, B. LaRose, R. D. Aquila, and B. Daryanian, "The grid of the future: Ten trends that will shape the grid over the next decade," IEEE Power Energy Mag., vol. 12, no. 3, pp. 26-36, May 2014.

[2] A. Golshani, W. Sun, and K. Sun, "An advanced power system partitioning method for fast and reliable restoration: Towards a self-healing power grid," IET Gener., Transm. Distrib., vol. 12, no. 1, Feb. 2018.

[3] F. Shen, Q. Wu, S. Huang, J. C. López, C. Li, and B. Zhou, "Review of service restoration methods distribution networks," in IEEE PES Innovative Smart Grid Technologies Conference Europe, Sarajevo, BH, pp. 1-6, Oct. 2018.

[4] Z. Wang and J. Wang, "Self-healing resilient distribution systems based on sectionalization into micro-grids," IEEE Trans. Power Syst., vol. 30, no. 6, pp. 3139-3149, Nov. 2015.

[5] C. Chen, C. Lin, and H. Tsai, "A rule-based expert system with colored petri net models for distribution system service restoration," IEEE Trans. Power Syst., vol. 17, no. 4, pp. 1073-1080, Nov. 2002.

[6] S. J. Lee, K. H. Kim, H. Y. Kim, J. K. Lee, and K. Y. Nam, “Expert system-aided service restoration in distribution automation," In Proceeding of IEEE International Conference System, Man and Cybernetics, Chicago, IL, USA, pp. 157-161, Oct. 1992.

[7] M. R. Kleinberg, K. Miu, and H. D. Chiang, "Improving service restoration of power distribution systems through load curtailment of in-service customers," IEEE Trans. Power Syst., vol. 26, no. 3, pp. 1110-1117, Aug. 2011.

[8] A. Botea, J. Rintanen, and D. Banerjee, "Optimal reconfiguration for supply restoration with informed Aast search," IEEE Trans. Smart Grid, vol. 3, no. 2, pp. 583-593, Jun. 2012.

[9] T. Nagata and H. Sasaki, “A multi-agent approach to power system restoration,” IEEE Trans. Power Syst., vol. 17, no. 2, pp. 457-462, May 2002.

[10]C. P. Nguyen and A. J. Flueck, “Agent based restoration with distributed energy storage support in smart grids," IEEE Trans. Smart Grid, vol. 3, no. 2, pp. 1029-1038, May 2012.

[11] A. Sharma, D. Srinivasan, and A. Trivedi, “A decentralized multi-agent system approach for service restoration using DG islanding," IEEE Trans. Smart Grid, vol. 6, no. 6, pp. 2784-2793, Nov. 2015.

[12] A. Sharma, D. Srinivasan, and A. Trivedi, “A decentralized multi-agent system approach for service restoration in uncertain environment," IEEE Trans. Smart Grid, vol. 9, no. 4, pp. 3394-3405, Nov. 2018.

[13] Y. T. Hsiao and C. Y. Chien, "Enhancement of restoration service in distribution systems using a combination fuzzy-GA method," IEEE Trans. Power Syst., vol. 15, no. 4, pp. 1394-1400, Nov. 2000.

[14]W. P. Luan, M. R. Irving, and J. S. Daniel, "Genetic algorithm for supply restoration and optimal load shedding in power system distribution networks," in Proceeding of IEE Generation, Transmission and Distribution, vol. 149, no. 2, pp. 145-151, Mar. 2002.

[15] J. Li, X. Y. Ma, C. C. Liu, and K. P. Schneider, "Distribution system restoration with micro-grids using spanning tree search," IEEE Trans. Power Syst., vol. 29, no. 6, pp. 3021-3029, Nov. 2014. 
[16] M. Zadsar, M. R. Haghifam, and S. M. M. Larimi, “Approach for self-healing resilient operation of active distribution network with micro-grid,” IET Gener., Transm. Distrib., vol. 11, no. 18, pp. 4633-4643, Dec. 2017.

[17] S. Khushalani, J. M. Solanki, and N. N. Schulz, “Optimized restoration of unbalanced distribution systems," IEEE Trans. Power Syst., vol. 22, no. 2, pp. 624630, May 2017.

[18]P. L. Cavalcante, J. F. F. J. C. López, M. J. Rider, A. V. Garcia, M. R. R. Malveira, L. L. Martins, and L. c. M. Direito, "Centralized self-healing scheme for electrical distribution systems," IEEE Trans. Smart Grid, vol. 7, no. 1, pp. 145-155, Jan. 2016.

[19] J. C. López, J. F. Franco, M. J. Rider, and R. Romero, "Optimal restoration/maintenance switching sequence of unbalanced three-phase distribution systems," IEEE Trans. Smart Grid, vol. 9, no. 6, pp. 6058-6068, Nov. 2018.

[20]B. Chen, C. Chen, J. Wang, and K. L. Butler-Purry, "Multi-time step service restoration for advanced distribution systems and micro-grids," IEEE Trans. Smart Grid, vol. 9, no. 6, pp. 6793-6805, Nov. 2018.

[21]X. Chen, W. Wu, and B. Zhang, "Robust restoration method for active distribution networks," IEEE Trans. Power Syst., vol. 31, no. 5, pp. 4005-4015, Sep. 2016.

[22]K. Chen, W. Wu, B. Zhang, and H. Sun, "Robust restoration decision making model for distribution networks based on information gap decision theory," IEEE Trans. Smart Grid, vol. 6, no. 2, pp. 587-597, Mar. 2015.

[23]R. R. Nejad and W. Sun, "Distributed load restoration in unbalanced active distribution systems", IEEE Trans. Smart Grid, in press.

[24] M. Lavorato, J. F. Franco, M. J. Rider, and R. Romero, "Imposing radiality constraints in distribution system optimization problems," IEEE Trans. Power Syst., vol. 27, no. 1, pp. 172-180, Feb. 2012.

[25] W. Zheng, W. Wu, B. Zhang, Z. Li, and Y. Liu, "Fully distributed multi-area economic dispatch method for active distribution networks," IET Gener., Transm. Distrib., vol. 9, no. 12, pp. 1341-1351, Aug. 2015.

[26] W. Zheng, W. Wu, B. Zhang, and C. Lin, "Distributed optimal residential demand response considering operational constraints of unbalanced distribution networks," IET Gener., Transm. Distrib., vol. 12, no. 9, pp. 1970-1979, May 2018.

[27] S. Huang, Q. Wu, Y. Guo, X. Chen, B. Zhou, and C. Li, "Distributed voltage control based ADMM for large-scale wind farm cluster connected to VSCHVDC," IEEE Trans. Sustain. Energy, in press.

[28] S. Boyd, N. Parikh, E. Chu, B. Peleato, and J. Eckstein, "Distributed optimization and statistical learning via the alternating direction method of multipliers," Foundations and Trends in Machine Learning, vol. 3, no. 1, pp. 1-122, Jan. 2010.

[29]J. Jian, C. Zhang, L. Yang, and K. Meng, "A hierarchical alternating direction method of multipliers for fully distributed unit commitment," International Journal of Electrical Power and Energy, in press.

[30] W. Zheng, W. Wu, B. Zhang, H. Sun, and Y. Liu, "A fully distributed reactive power optimization and control method for active distribution networks," IEEE Trans. Smart Grid, vol. 7, no. 2, pp. 1021-1033, Mar 2016.

[31] IEEE Power and Energy Society (PES). Distribution Test Feeders: 123- bus Feeder [Online]. Available: http://ewh.ieee.org/soc/pes/dsacom/testfeeders/

[32]948 buses test system. https://www.feis.unesp.br/?fbclid=IwAR3zeXtdZIESqGW0xGvz5vOrRnJetaJNPuSZfogv5-bMvRhbZl-sivufWFo\#!/departamentos/engenharia-eletrica/pesquisas-e-projetos/lapsee/downloads/materiais-de-cursos1193/ 